\title{
REVIEWS
}

\section{The Basic Conservative Treatment of Temporomandibular Joint Anterior Disc Displacement Without Reduction - Review}

\author{
Department of Prosthetic Dentistry, Wroclaw Medical University, Poland
}

A - research concept and design; $\mathbf{B}$ - collection and/or assembly of data; $\mathbf{C}$ - data analysis and interpretation;

$\mathbf{D}$ - writing the article; $\mathbf{E}$ - critical revision of the article; $\mathbf{F}$ - final approval of article; $\mathbf{G}$ - other

\begin{abstract}
Temporomandibular joint derangement is a common disorder of the stomatognathic system. One type of these disorders is disc displacement without reduction with limited mouth opening, characterized by pain in affected TMJ and a decreased range of mouth opening. The natural course of closed lock is self-limiting. However, if there is no significant improvement after 12 weeks of following natural course of disc displacement without reduction, this is an indication that the therapy should be implemented. The article presents a review of the most commonly applied methods of basic conservative closed lock treatment. The most commonly applied methods of closed lock conservative management are: education and counseling, mandibular manipulation, splint therapy, exercise therapy and pharmacotherapy. The first choice method of treatment should be minimally invasive. The surgical management should be considered after unsuccessful conservative therapy. All presented methods of closed lock treatment seem to be effective in decreasing pain and reestablishing physiological range of motion (Adv Clin Exp Med 2015, 24, 4, 731-735).
\end{abstract}

Key words: TMD, closed lock, conservative treatment.

Internal derangement in temporomandibular joint (TMJ) is a common dysfunction of the stomatognathic system. According to classification of Research Diagnostic Criteria for TMD there are 3 main types of internal TMJ derangement: disc displacement with reduction and disc displacement without reduction with or without limited mouth opening. The prevalence of disc displacement is about $41 \%$ in TMD patients. The most common type is disc displacement with reduction characterized by clicking in the temporomandibular joint. In about $5 \%$ of cases the disc displacement without reduction with limited mouth opening is observed. The main symptoms of closed lock are pain in affected joint and decreased range of mouth opening $(\leq 35 \mathrm{~mm}$ ), which deteriorate the function of the stomatognathic system and force the patient to look for professional help [1-3].

The symptoms of closed lock are usually related to disc displacement without reduction. The etiology of disc displacement is not clear, but there are a few possible reasons playing a key role in changes of the TMJ structure, such as parafunctions (e.g. bruxism), anatomical factors, trauma or general hypermobility of the joints [4-7]. In the majority of cases the disc is displaced anteriorly, blocking mechanically translating movement of the condyle, resulting in restricted mouth opening. In addition the bilaminar zone becomes overloaded by direct contact with the condyle, which is the main reason of pain in the TMJ [8].

It has been stated that the natural course of disc displacement without reduction is self-limiting. Significant improvement of clinical symptoms of closed lock is observed in $75 \%$ of patients in a 2.5 year follow-up. The MRI scans reveal permanently displaced and deformed disc in untreated closed lock patients. The reduction of restriction of mouth opening and pain is most probably caused by active adaptation to new structural situation in TMJ [9-11]. Though anterior disc displacement without reduction is a self-limiting, 
benign condition, it is also well-known that internal derangement in TMJ may lead to osteoarthrosis [12]. Accordingly, if there is no significant improvement after 12 weeks of following natural course of disc displacement without reduction, treatment should be implemented. The initially applied therapy should be minimally invasive. The surgical methods of treatment should be considered after 6 months of unsuccessful conservative management [13-15].

\section{Conservative Therapy of Closed Lock}

\section{Education and Counseling}

As the natural course of closed lock is self-limiting, some authors recommend just education and counseling. It is important to explain the mechanism of disc displacement and the negative effects of parafunctions on the stomatognatic system, especially the TMJ. The recommended self-care protocol might include thermotherapy (cold or warm packs), soft food diet and gentle mouth-opening exercises in the initial period of locking. According to Minakuchi et al. and Craane et al. there is no significant difference in outcomes of self-care protocol, pharmacotherapy, splint therapy vs. no treatment, which might indicate that conducting treatment in closed lock patients is not necessary $[16,17]$.

\section{Mandibular Manipulation}

The aim of mandibular manipulation is to recapture a displaced disc. The common technique for mandibular manipulation is pulling the condyle of the affected side downward and forward in order to locate the condyle on the anteriorly displaced disc. Usually the operator's thumb is placed on occlusal surfaces of lower molars on the affected side and the rest of the fingers are covering the external surface of mandible. The other operator's hand is placed in temporal area to stabilize patients head $[18,19]$. According to Foster et al. this mandibular manipulation should be applied to all patients with symptoms of closed lock [20]. It is difficult to predict the therapeutic effect of this method as it depends not on the duration of locking, but rather on the stage of internal derangement. In cases of disc deformation manipulation brings no improvement. Kurita et al. reported that in $9 \%$ of cases, closed locked disc could be reduced by manipulation technique. If the procedure is performed under general anesthesia in the presence of muscle relaxants the percentage of successful outcomes rises up to $42 \%$ [19]. It was reported by Chiba et al. that the successful mandibular manipulation prevents progressive changes in the TMJ [21]. However, some authors question mandibular manipulation as an effective method of disc displacement without reduction treatment as it is not effective in most of the closed lock cases [22]. In cases of successful disc recapturing by manipulation technique, it was already recommended by Farrar that the occlusal splint should be applied immediately for the retention of the condyle-disc position [23].

The disc recapturing might be achieved not just by mandibular manipulation performed by the professional operator, but also by active repositioning of the disc done by the patient. The protocol of active manipulation described by Okeson consists of few mandibular movements: lateral movement from central occlusal position towards non-affected side, wide mouth opening and finally, closing the mouth to previous lateral position. The protocol can be repeated few times during one visit. According to Pihut et al. the unlocking of the TMJ disc can be achieved in $71.8 \%$ of patients who performed active recapturing exercises. After successful unlocking it is recommended that the patient is provided with an anterior repositioning splint in order to eliminate acoustic symptoms in TMJ [24].

\section{Splint Therapy}

The presence of any occlusal appliance leads to stress reduction in the temporomandibular joint secondary to change in the position of the mandible and decreased muscle activity. There are 3 types of occlusal appliances commonly used in the therapy of displaced disc without reduction: stabilization, distraction and anterior repositioning splints.

Stabilization splint slightly increases the vertical occlusal dimension. The occlusal contacts are present bilaterally on the flat surface of the splint. According to many authors, stabilization splint leads to a significant reduction of symptoms of closed lock $[25,26]$. The distraction splint provides greater articular stress release. The occlusal contacts are located mostly in the posterior part of the splint. Both splints cause distraction in TMJ, but in the case of distraction splint this effect is intensified. Schmitter et al. reported that stabilization splint seems to be more effective than distraction splint in closed lock therapy [27]. The result of splint therapy is achieved not by improving the disc position, but is strongly related to the basic decoupling of neuromuscular reflex mechanism 
and reduction of TMJ stress $[28,29]$. The recommended time of splint usage, regardless type of the splint, is day and night, excluding the mealtimes and teeth brushing [28]. However, Haketa et al. recommended wearing a stabilization splint to closed lock patients just during the night rest [25]. Stiesch-Scholz et al. concluded that stabilization splint therapy is an efficient method of closed lock treatment. In that study the significant improvement of clinical outcomes was present in $92.7 \%$ of patients [30]. Other authors have reported less spectacular results [31,32]. According to Lundh et al. the therapeutic effect of flat occlusal splints was observed in $32 \%$ of patients. The same authors presented evidence that there is no significant difference between outcomes of flat splint therapy and nontreatment group of patients [33].

The aim of anterior repositioning splint is to reestablish physiological disc-condyle relationship. However, the stability of disc recapturing depends on the range of disc displacement and the stage of internal dearangement in TMJ. In cases of persistent closed lock protrusive occlusal splint does not lead to recapturing the disc, but has a pain-relieving effect $[34,35]$.

\section{Exercise Therapy}

One of the methods of physical therapy in cases of anterior displacement of the disc without reduction is exercise therapy, including active and passive movements of the mandible. Active exercises are used to correct the mouth opening path. Passive exercises improve mouth opening range [36]. According to Nicolakis et al., exercise therapy reduces pain in $80 \%$ of cases and improves the range of motion in $75 \%$ of closed lock patients. Those results indicate that exercise therapy is significantly more effective than no therapy [37].

The protocol of Mandibular Condylar Movement Exercise (MCME) presented by Yoshida $\mathrm{H}$ et al. seems to be easy to perform in every day clinical practice and as a home exercise for patients. It should be repeated 8 times per day. The first part of the exercise consists of active movements, which are repeated during a $10-\mathrm{min}$ period: lateral motion towards right and left side, protrusion and wide mouth opening. The second part of the MCME is the passive exercise - opening mouth by pushing simultaneously the thumb against the upper anterior teeth and forefinger against the lower anterior teeth. It was reported that this algorithm of exercise therapy significantly increases the range of mouth opening in closed lock cases. Also the protrusion and lateral movements improve after performing MCME. In addition, it was stated that the effectiveness of that therapy depends on the age and duration of locking $[38,39]$.

Similar stretching exercise was conducted in research of Haketa et al. Closed lock patients performed manual jaw opening exercise set 4 times per day during 8 -week therapy. The single set included 3 cycles of stretching, each cycle lasted $30 \mathrm{~s}$. The effect of this exercise therapy on mouth-opening range was significantly better than occlusal splint usage. The reason for the increased range of motion was due to the stretching of the posterior disc attachment leading to more anterior disc location [25].

The research of Muhtaroğullari et al. was based on the performed exercise therapy with the simultaneous use of a stabilization splint in those cases where manual manipulation of anteriorly displaced disc failed. The splint was worn day and night, excluding the mealtimes. The stretching exercises - opening mouth and lateral movements were performed 5 times per day for $5 \mathrm{~min}$. After a 6-month period of treatment all patients reported a beneficial effect of the therapy. However, the MRI showed that this success is not related to improved TMJ morphology [40].

\section{Pharmacotherapy}

The most commonly used medicaments in management of anterior disc displacement without reduction are non-steroidal anti-inflammatory drugs (NSAIDS), which have an anti-inflammatory and analgesic effect [29]. Schiffman et al. recommended ibuprofen in a dose of $800 \mathrm{mg}, 3$ times per day up to 6 weeks of treatment [14]. Minakuchi et al. used diclofenac sodium in doses of $25 \mathrm{mg}$, 3 times per day up to 8 weeks. Moreover, Minakuchi et al. emphasized the need of an anti-gastric ulcer medication during NSAIDS therapy [16]. In cases of marked muscle tension muscle relaxants might be prescribed for the initial 1-2 weeks of treatment. The medication used can be cyclobenzaprine in dose of $10 \mathrm{mg}$, once a day, at bedtime [14].

\section{Conclusions}

The anterior disc displacement without reduction is a benign, self-limiting condition. As the first step of treatment the mandibular manipulation is advised in order to recapture the displaced disc. In cases of successful mandibular manipulation, the patient should be provided with occlusal appliance. If mandibular manipulation fails, the patient should be educated about the TMJ condition. At that time performing conservative treatment is 
not necessary, as the clinical symptoms of closed lock usually decrease in time. In cases of lack of self-improvement, any means of conservative treatment should be applied. According to the clinical experience of authors, exercise therapy in addition to education and counseling are considered the most efficient methods of anterior disc displacement without reduction conservative therapy.

\section{References}

[1] Dworkin SF, LeResche L: Research diagnostic criteria for temporomandibular disorders: review, criteria, examinations and specifications, critique. J Craniomandib Disord 1992, 6, 301-355.

[2] Manfredini D, Guarda-Nardini L, Wincour E, Piccotti F, Ahlberg J, Lobbezoo F: Research diagnostic criteria for temporomandibular disorders: a systematic review of axis I epidemiologic findings. Oral Surg Oral Med Oral Pathol Oral Radiol Endod 2011, 112, 453-462.

[3] Peroz I, Tai S: Masticatory performance in patients with anterior disc displacement without reduction in comparison with symptoms-free volunteers. Eur J Oral Sci 2002, 110, 341-344.

[4] Katzberg RW, Westesson PL, Tallents RH, Drake CM: Anatomic disoredrs of the temporomandibular joint disc in asymptomatic subjects. J Oral Maxillofac Surg 1996, 54, 147-153.

[5] Wongwatana S, Kronman JH, Clark RE, Kabani S, Mehta N: Anatomic basis for disk displacement in temporomandibular joint (TMJ) dysfunction. Am J Orthod Dentofacial Orthop 1994, 105, 257-264.

[6] Kavuncu V, Sahin S, Kamanli A, Karan A, Aksoy C: The role of systemic hypermobility and condylar hypermobility in temporomandibular joint dysfunction syndrome. Rheumatol Int 2006, 26, 257-260.

[7] Garcia R Jr, Arrington JA: The relationship between cervical whiplash and temporomandibular joint injuries: an MRI study. Cranio 1996, 14, 233-239.

[8] Pérez del Palomar A, Doblaré M: An accurate simulation model of anteriorly displaced TMJ discs with and without reduction. Med Eng Phys 2007, 29, 216-226.

[9] Kurita K, Westesson PL, Yuasa H, Toyama M, Machida J, Ogi N: Natural course of untreated symptomatic temporomandibular joint disc displacement without reduction. J Dent Res 1998, 77, 361-365.

[10] Imirzalioglu P, Biler N, Agildere AM: Clinical and radiological follow-up results of patients with untreated TMJ closed lock. J Oral Rehabil 2005, 32, 326-331.

[11] Cai XY, Jin JM, Yang C: Changes in disc position, disc length, and condylar height in the temporomandibular joint with anterior disc displacement: a longitudinal retrospective magnetic resonance imaging study. J Oral Maxillofac Surg 2011, 69, 340-346.

[12] Dimitroulis G: The prevalence of osteoarthrosis in cases of advanced internal derangement of the temporomandibular joint: a clinical, surgical and histological study. Int J Oral Maxillofac Surg 2005, 34, 345-349.

[13] Yura S: Natural course of acute closed lock of the temporomandibular joint. Br J Oral Maxillofac Surg 2012, 50, 646-649.

[14] Schiffman EL, Velly AM, Look JO, Hodges JS, Swift JQ, Decker KL, Anderson QN, Templeton RB, Lenton PA, Kang W, Fricton JR: Effects of four treatment strategies for temporomandibular joint closed lock. Int J Oral Maxillofac Surg 2014, 43, 217-226.

[15] Schiffman EL, Look JO, Hodges JS, Swift JQ, Decker KL, Hathaway KM, Templeton RB, Fricton JR: Randomized effectiveness study of four therapeutic strategies for TMJ closed lock. J Dent Res 2007, 86, 58-63.

[16] Minakuchi H, Kuboki T, Matsuka Y, Maekawa K, Yatani H, Yamashita A: Randomized controlled evaluation of non-surgical treatments for temporomandibular joint anterior disk displacement without reduction. J Dent Res 2001, 80, 924-928.

[17] Craane B, Dijkstra PU, Stappaerts K, De Laat A: Randomized controlled trial on physical therapy for TMJ closed lock. J Dent Res 2012, 91, 364-369.

[18] Jagger RG: Mandibular manipulation of anterior disc displacement without reduction. J Oral Rehabil 1991, 18, 497-500.

[19] Kurita H, Kurashina K, Ohtsuka A: Efficacy of a mandibular manipulation technique in reducing the permanently displaced temporomandibular joint disc. J Oral Maxillofac Surg 1999, 57, 784-787.

[20] Foster ME, Gray RJ, Davies SJ, Macfarlane TV: Therapeutic manipulation of the temporomandibular joint. Br J Oral Maxillofac Surg 2000, 38, 641-644.

[21] Chiba M, Echigo S: Longitudinal MRI follow-up of temporomandibular joint internal derangement with closed lock after successful disk reduction with mandibular manipulation. Dentomaxillofac Radiol 2005, 34, 106-111.

[22] Alves BM, Macedo CR, Januzzi E, Grossmann E, Atallah AN, Peccin S: Mandibular manipulation for the treatment of temporomandibular disorder. J Craniofac Surg 2013, 24, 488-493.

[23] Farrar WB: Characteristics of the condylar path in internal derangements of the TMJ. J Prosthet Dent 1978, 39, 319-323.

[24] Pihut M, Wiśniewska G, Majewski S: Active repositioning of temporomandibular disc displacement without reduction. J Stoma, 2013, 66, 650-662.

[25] Haketa T, Kino K, Sugisaki M, Takaoka M, Ohta T: Randomized clinical trial of treatment for TMJ disc displacement. J Dent Res 2010, 89, 1259-1263.

[26] Shoji YN: Nonsurgical treatment of anterior disk displacement of the temporomandibular joint: a case report on the relationship between condylar rotation and translation. Cranio 1995, 13, 270-273. 
[27] Schmitter M, Zahran M, Duc JM, Henschel V, Rammelsberg P: Conservative therapy in patients with anterior disc displacement without reduction using 2 common splints: a randomized clinical trial. J Oral Maxillofac Surg 2005, 63, 1295-1303.

[28] Stiesch-Scholz M, Kempert J, Wolter S, Tschernitschek H, Rossbach A: Comparative study on splint therapy of anterior disc displacement without reduction. J Oral Rehabil 2005, 32, 474-479.

[29] Ohnuki T, Fukuda M, Nakata A, Nagai H, Takahashi T, Sasano T, Miyamoto Y: Evaluation of the position, mobility, and morphology of the disc by MRI before and after four different treatments for temporomandibular joint disorders. Dentomaxillofac Radiol 2006, 35, 103-109.

[30] Stiesch-Scholz M, Tschernitschek H, Rossbach A: Early begin of splint therapy improves treatment outcome in patients with temporomandibular joint disk displacement without reduction. Clin Oral Investig 2002, 6, 119-123.

[31] Lee HS, Baek HS, Song DS., Kim HC, Kim HG, Kim BJ, Kim MS, Shin SH, Jung SH, Kim CH: Effect of simultaneous therapy of arthrocentesis and occlusal splints on temporomandibular disorders: anterior disc displacement without reduction. J Korean Assoc Oral Maxillofac Surg 2013, 39, 14-20.

[32] Kai S, Kai H, Tabata O, Shiratsuchi Y, Ohishi M: Long-term outcomes of nonsurgical treatment in nonreducing anteriorly displaced disk of the temporomandibular joint. Oral Surg Oral Med Oral Pathol Oral Radiol Endod $1998,85,258-267$.

[33] Lundh H, Westesson PL, Eriksson L, Brooks SL: Temporomandibular joint disk displacement without reduction. Oral Surg Oral Med Oral Pathol 1992, 73, 655-658.

[34] Eberhard D, Bantleon HP, Steger W: The efficacy of anterior repositioning splint therapy studied by magnetic resonance imaging. Eur J Orthod 2002, 24, 343-352.

[35] Simmons HC $3^{\text {rd }}$, Gibbs SJ: Recapture of temporomandibular joint disks using anterior repositioning appliances: an MRI study. Cranio 1995, 13, 227-237.

[36] Komatsu K, Yamaguchi T, Ohata N: A new training device for rehabilitation of lateral mandibular movements: a pilot study. Cranio 2002, 20, 198-203.

[37] Nicolakis P, Erdogmus B, Kopf A, Ebenbichler G, Kollmitzer J, Piehslinger E, Fialka-Moser V: Effectiveness of exercise therapy in patients with internal derangement of the temporomandibular joint. J Oral Rehabil 2001, 28, $1158-1164$

[38] Yoshida H, Kashiwagi K, Sakata T, Tanaka M, Kawazoe T, Morita S: Prognostic factor of mandibular condylar movement exercise for patients with internal derangement of the temporomandibular joint on initial presentation: Preliminary report. J Craniomanillofac Surg 2013, 41, 356-358.

[39] Yoshida H, Sakata T, Hayashi T, Shirao K, Oshiro N, Morita S: Evaluation of mandibular condylar movement exercise for patients with internal derangement of the temporomandibular joint on initial presentation. Br J Oral Maxillofac Surg 2011, 49, 310-313.

[40] Muhtaroğullari M, Ertan AA, Demiralp B, Canay S: Correlation between clinical and magnetic resonance imaging findings in the treatment of anterior disc displacement. Int J Prosthodont 2013, 26, 138-142.

\section{Address for correspondence:}

Marta Miernik

Department of Prosthetic Dentistry

Wroclaw Medical University

Krakowska 26

50-425 Wrocław

Poland

E-mail: marta_miernik@onet.eu

Conflict of interest: None declared

Received: 7.09.2014

Revised: 19.11.2014

Accepted: 23.03.2015 